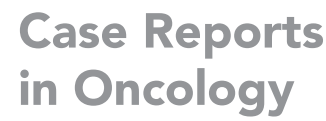

Case Reports

in Oncology

\title{
Sarcoma of the Heart Treated with Stereotactic MR-Guided Online Adaptive Radiation Therapy
}

\author{
Jacquelien Pomp ${ }^{a} \quad$ Bram van Asselen ${ }^{a}$ Robbert H.A. Tersteeg ${ }^{a}$ \\ Aryan Vink $^{b}$ Rutger J. Hassink ${ }^{c}$ Niels P. van der Kaaij ${ }^{d}$ \\ Guido E.E.H.L. van Aarnhem ${ }^{d}$ Joost J.C. Verhoeffa \\ aDepartment of Radiation Oncology, University Medical Center Utrecht, Utrecht, \\ The Netherlands; 'bepartment of Pathology, University Medical Center Utrecht, Utrecht, \\ The Netherlands; 'Department of Cardiology, University Medical Center Utrecht, Utrecht, \\ The Netherlands; ${ }^{d}$ Department of Cardiothoracic Surgery, University Medical Center \\ Utrecht, Utrecht, The Netherlands
}

\section{Keywords}

Heart · Intimal sarcoma - Stereotactic body radiation therapy · Stereotactic MR-guided online adaptive radiation therapy $\cdot$ MR-Linac

\section{Abstract}

We present the first case in the literature of a patient with a histology-proven intimal sarcoma of the heart, recurrent after surgery, treated with stereotactic MR-guided online adaptive radiation therapy on an MR-Linac machine. The treatment was feasible and well tolerated. The CT scan 6 months after the last treatment showed stable disease.

\section{Introduction}

The MR-Linac system is the latest equipment in radiotherapy: it integrates an MRI scanner inside a linear accelerator, allowing it to obtain MR images during treatment. As conventional accelerators mostly have an integrated CT scanner for online imaging, soft tissues are not as well visible as on an MRI. We present a case of recurrent inoperable intimal sarcoma of the heart treated with MR-guided radiotherapy. 


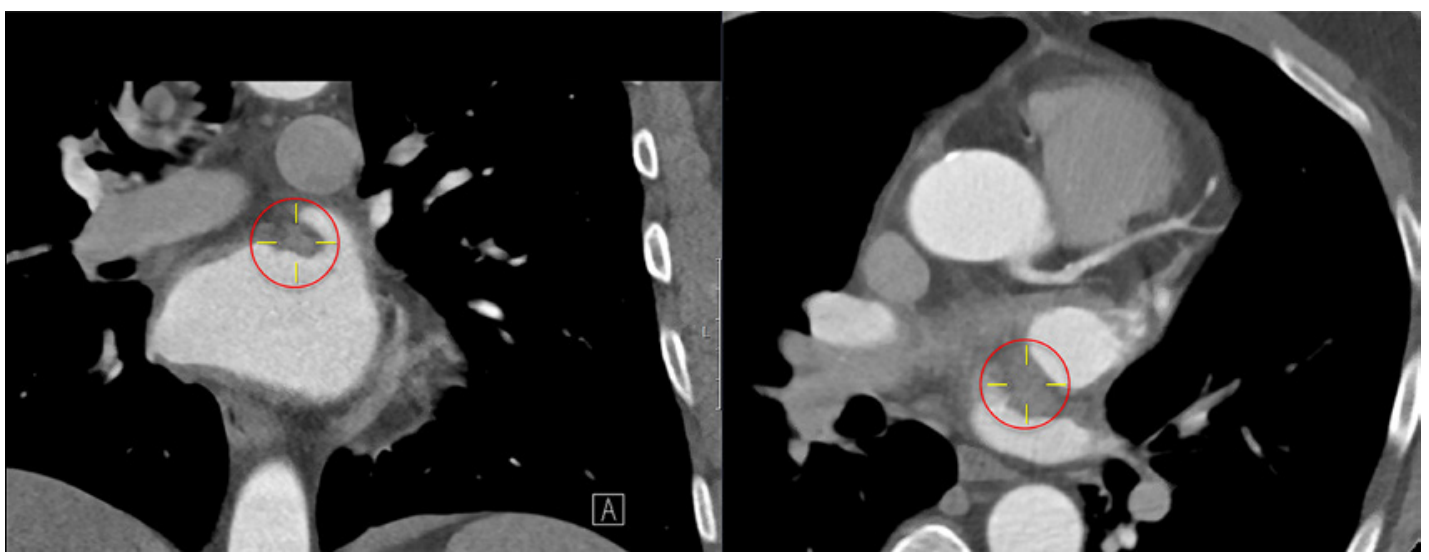

Fig. 1. Preoperative diagnostic contrast-enhanced CT scan, transversal and coronal view. The tumour is visualised by yellow crosshairs.

\section{Case Presentation}

A 58-year-old man was referred to the ER of our hospital in March 2019, having a cerebral vascular accident. His medical history reported high blood pressure and hypercholesterolemia. He had quit smoking in 2018 (40 pack-years).

Despite initiation of anticoagulants, he suffered from recurrent strokes, and in May 2019, a cardiology work-up CT scan revealed a lobular mass located in the left atrium of the heart. The tumour rather resembled a cardiac myxoma than a thrombus and was best visible on a contrast CT scan and ultrasound (Fig. 1).

Cardiac surgery was performed, and a mucinous tumour, attached to the wall of the left atrium and left lower pulmonary vein, was removed. The tumour could easily be excised, and frozen sections were not conclusive. The resection was macroscopically complete.

Definitive histology showed a lesion at the transition of the left lower pulmonary vein to the left atrium consisting of a proliferation of mesenchymal cells with moderate cytonuclear atypia and varying cellularity. Part of the lesion protruded into the lumen, and part of the lesion showed an invasive growth pattern. CDK4+ and MDM2+ amplification, as determined by multiplex ligation-dependent probe amplification, was positive, which is characteristic of an intimal sarcoma, a very rare tumour belonging to the class of malignant soft tissue sarcomas. Microscopically, the resection margins contained tumour tissue, so the resection was considered incomplete.

A PET-CT scan for staging did not show any metastases. No adjuvant systemic treatment was given, and the patient remained symptom free until November 2019, when he had another stroke. On the CT scan, a small lesion at the base of the resection area was seen, suspicious for local recurrence (Fig. 2). As re-surgery was not an option, after multidisciplinary board discussions, the patient was referred to the radiotherapy department. It was decided to irradiate the tumour with stereotactic MR-guided online adaptive radiation therapy (SMART), to a high dose, i.e. 60 Gy in 12 fractions during a 4-week overall treatment time.

Treatment preparation started with delineation of the tumour (visible with contrast) and surrounding tissues on a planning CT scan. The heart, lungs, trachea, bronchi and oesophagus were contoured as organs at risk. The total prescribed dose could be delivered while keeping the dose to the lungs, trachea, bronchi and oesophagus below the tolerance dose. Under- 


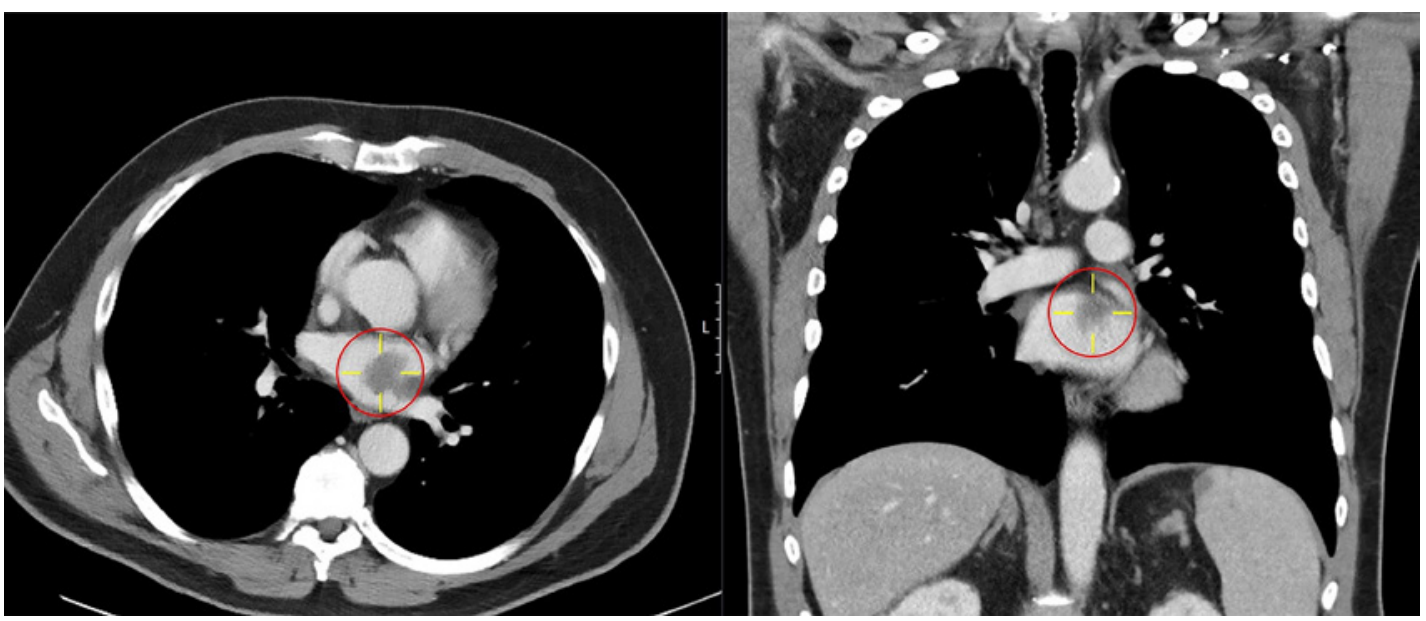

Fig. 2. Recurrent disease on contrast-enhanced CT, November 2019. The tumour is visualised by yellow crosshairs.

standably, due to the location of the tumour, the dose constraints could not be met for the heart. As conventional linear accelerators have an integrated CT scan for online imaging, it was suggested that on a cone beam CT (CBCT) it would not be possible to adequately visualize the tumour location and the different parts of the heart due to poor image contrast. Therefore, the patient was treated on an MR-Linac system. To prepare for treatment on the MR-Linac, the original planning CT scan was deformably registered by T2-weighted 3D MRI. The patient underwent SMART (online suppl. Video; see www.karger.com/doi/10.1159/000513623 for all online suppl. material) 12 times at 5 Gy with daily plan adaptation, which he uneventfully completed on February 12, 2020. The third follow-up CT scan, made 6 months after the last treatment, in August 2020, showed stable disease.

\section{Discussion and Conclusion}

Intimal sarcoma is defined as a malignant tumour arising in the tunica intima of large blood vessels. It is a rare malignant mesenchymal tumour, belonging to the group of soft tissue sarcomas, and has a poor prognosis. Two case reports described the rapidly fatal outcome of this sarcoma $[1,2]$. In a review paper, amongst 13 patients with primary angiosarcomas originating from the heart, aorta or great vessels, 10 patients had distant metastases, mostly to the lungs [3]. Of 212 cases of cardiac tumours reviewed by Wang et al. [4], $85 \%$ were cardiac myxomas, $15 \%$ were non-myxomas, and only $6 \%$ were malignant tumours. All primary cardiac sarcomas recurred. Barreiro et al. [5] described 73 cases of cardiac tumours, of which $15 \%$ proved to be malignant and only 4 were undifferentiated sarcomas with an $80 \%$ mortality rate at 1 year.

MDM2 amplification, determined by immunohistochemistry and by fluorescence and dual-colour in situ hybridization, is characteristic of this malignant sarcoma of the intima, and can help to distinguish it from angiosarcoma [6, 7]. Cardiac MRI is the standard diagnostic modality for the differentiation and characterization of cardiac masses, although histopathological confirmation remains the preferred method, especially now that there are more personalized treatment options, even for tumours of the heart. Therefore, in cardio-oncology, imaging becomes more important [8]. The addition of FDG-PET to the MR scan may increase 
Table 1. Calculated $\mathrm{BED}$ and $\mathrm{EQD}_{2}$ for $12 \times 5$ Gy and $25 \times 2$ Gy schemes

\begin{tabular}{lllll}
\hline Dose fractionation & $\operatorname{BED}(\alpha / \beta=3)$ & $\operatorname{BED}(\alpha / \beta=10)$ & $\mathrm{EQD}_{2}(\alpha / \beta=3)$ & $\mathrm{EQD}_{2}(\alpha / \beta=10)$ \\
\hline $12 \times 5$ Gy & 160 & 90 & 96 & 75 \\
$25 \times 2$ Gy & 83 & 60 & 50 & 50 \\
\hline
\end{tabular}

$\mathrm{BED}$, biological effective dose; $\mathrm{EQD}_{2}$, equivalent dose for 2 Gy fractions.

the diagnostic potential [9]. An approach less invasive than open heart surgery to diagnose a cardiac tumour may be ultrasound or CT scanning [10].

Complete surgical resection is the first choice, but this is often not possible due to the surrounding critical structures, and therefore local recurrence frequently occurs $[1,11]$. Definitive radiotherapy is uncommonly used in the management of soft tissue sarcoma. It is only considered in irresectable cases, and a high dose of radiation is needed to obtain local control [12]. Very few data exist on the use of radiotherapy for sarcomas of the heart [13].

Treatment of tumours located near or in the heart is challenging both with regard to the surgical and the radiotherapeutical approach. Our patient underwent surgical intervention, which allowed obtaining tissue for diagnosis. When the tumour recurred, it was decided to try to give a radical radiation dose locally.

Regarding radiotherapy of locations in or near the heart, there are several obstacles to overcome. First, there is the movement of the mediastinum including the heart due to respiratory movement, and cardiac movement due to the heartbeat $[14,15]$. Second, there is the maximum tolerated dose to the heart and its nearest critical organs.

We realized that when treating the patient on a linear accelerator, equipped with CBCT, for image-guided radiotherapy, the image quality would be insufficient to discriminate structures of the heart, or the tumour itself. MRI, on the other hand, offers better soft tissue contrast than does CT or CBCT [16]. Furthermore, on the MR-Linac system, it is possible to adapt the plan daily to correct for position changes and deformations of the heart and surrounding healthy tissue. Therefore, it was decided to treat the patient on the MR-Linac, a 7-MV linear accelerator with an integrated 1.5-T MR scanner which can image the tumour and the moving normal surrounding tissues in real time during irradiation $[17,18]$.

An advantage of MRI during radiation treatment, i.e., MR-guided radiotherapy, has been acknowledged for areas that do not move too much, such as the brain, prostate, and rectum. However, there is the hurdle of visualizing moving structures in real time on so-called cine MRI [16]. Since the beginning of the 21st century, the concept of integrating an MR scanner with an accelerator has been developed at the University Medical Centre Utrecht (UMCU). Having started with blurry static images, it is now possible to treat moving targets via online imaging and adapt the plan to daily anatomic variation. The next step is to calculate dosedelivering and plan adaptation in real time [19]. The daily treatment procedure on an MR-Linac system takes longer than on conventional linear accelerators, but this is largely compensated by the use of hypofractionation, making it possible to deliver the total dose in fewer fractions. Furthermore, the biological effective dose and the equivalent dose for 2 Gy fractions are much higher with hypofractionated stereotactic body radiation therapy than with conventional fractionated radiation treatment (Table 1).

The cases of intimal sarcoma of the heart presented in the literature and treated with radiotherapy were mostly irradiated with 3D conformal radiotherapy in standard fractionation of 2 Gy per day for 25-30 fractions. To our knowledge, no data on stereotactic body radiation therapy with curative intent for cardiac sarcoma exist. 
In conclusion, we presented the first case of a recurrent intimal sarcoma of the heart treated with SMART on the MR-Linac system. The treatment was feasible and well tolerated.

\section{Statement of Ethics}

Written informed consent was obtained from the patient for publication of this case report and any accompanying images.

\section{Conflict of Interest Statement}

The authors have no conflicts of interest to declare.

\section{Funding Sources}

There was no funding provided for the writing of this manuscript.

\section{Author Contributions}

All authors equally contributed to writing and editing, and agreed on submission of the final version. J. Pomp wrote the first draft and made the layout, integrated comments from the other authors, and provided final approval and submission. B. van Asselen was responsible for the description workflow on the MR-Linac and technical details, and contributed by critically revising the paper. R.H.A. Tersteeg was the treating physician, and contributed by reading and revising the manuscript critically and advising on the patient workflow. A. Vink reviewed the slides and wrote the part about histology; he also reviewed the paper critically and made useful suggestions. R.J. Hassink diagnosed the patient, critically reviewed part with the patient's history, and reviewed the paper and gave useful comments. N.P. van der Kaaij performed the surgery together with G.E.E.H.L. van Aarnhem, revised the paper, and is responsible for the description of the surgery. G.E.E.H.L. van Aarnhem performed the surgery with N.P. van der Kaaij, revised the paper, and is responsible for the description of the surgery. J.J.C.Verhoeff initiated the paper, revised and commented on the later versions, and contributed the figures and video for this paper.

\section{References}

1 Thuraisingam A, Fletcher N, McKay G, Smiles J. Primary cardiac intimal sarcoma. Ann Surg Case Rep. 2019; 2(1):1013.

2 Ogechukwu C-N, Christopher N, Christoph S, Etinosasere O, Jose B. The insidious cardiac tumor: a primary left atrium intimal cardiac sarcoma in a young patient. Case Rep Cardiol. 2019;2019:7245676.

3 Fatima J, Duncan AA, Maleszewski JJ, Kalra M, Oderich GS, Gloviczki P, et al. Primary angiosarcoma of the aorta, great vessels, and the heart. J Vasc Surg. 2013;57(3):756-64.

4 Wang JG, Wang B, Hu Y, Liu JH, Liu B, Liu H, et al. Clinicopathologic features and outcomes of primary cardiac tumors: a 16-year-experience with 212 patients at a Chinese medical center. Cardiovasc Pathol. 2018 MarApr;33:45-54.

5 Barreiro M, Renilla A, Jimenez JM, Martin M, Al Musa T, Garcia L, et al. Primary cardiac tumors: 32 years of experience from a Spanish tertiary surgical center. Cardiovasc Pathol. 2013;22(6):424-7.

6 Grant L, Morgan I, Sumathi V, Salmons N. Intimal sarcoma of the left atrium presenting with transient ischaemic attack - a case report and review of the literature. J Cardiol Cases. 2019;21(3):89-92.

\section{Karger's}


7 Jimbo N, Komatsu M, Itoh T, Hirose T. MDM2 dual-color in situ hybridization (DISH) aids the diagnosis of intimal sarcomas. Cardiovasc Pathol. 2019 Nov-Dec;43:107142.

8 Steingart RM, Chandrashekhar Y, Marwick TH. Imaging in cardio-oncology: where are we and where should we be going? JACC Cardiovasc Imaging. 2018;11(8):1209-11.

9 Fathala A, Abouzied M, AlSugair AA. Cardiac and pericardial tumors: a potential application of positron emission tomography-magnetic resonance imaging. World J Cardiol. 2017;9(7):600.

10 Donisan T, Balanescu DV, Lopez-Mattei JC, Kim P, Leja MJ, Banchs J, et al. In search of a less invasive approach to cardiac tumor diagnosis: multimodality imaging assessment and biopsy. JACC Cardiovasc Imaging. 2018; 11(8):1191-5.

11 Li Z, Hsieh T, Salehi A. Recurrent cardiac intimal (spindle cell) sarcoma of the left atrium. J Cardiothorac Vasc Anesth. 2013;27(1):103-7.

12 Kepka L, DeLaney TF, Suit HD, Goldberg SI. Results of radiation therapy for unresected soft-tissue sarcomas. Int J Radiat Oncol Biol Phys. 2005;63(3):852-9.

13 Moeri-Schimmel R, Pras E, Desar I, Krol S, Braam P. Primary sarcoma of the heart: case report and literature review. J Cardiothorac Surg. 2020;15(1):104-6.

14 Seppenwoolde Y, Shirato H, Kitamura K, Shimizu S, van Herk M, Lebesque JV, et al. Precise and real-time measurement of 3D tumor motion in lung due to breathing and heartbeat, measured during radiotherapy. Int J Radiat Oncol Biol Phys. 2002;53(4):822-34.

15 Keall PJ, Mageras GS, Balter JM, Emery RS, Forster KM, Jiang SB, et al. The management of respiratory motion in radiation oncology report of AAPM Task Group 76. Med Phys. 2006 Oct;33(10):3874-900.

16 Menten MJ, Wetscherek A, Fast MF. MRI-guided lung SBRT: present and future developments. Phys Med. 2017; 44:139-49.

17 Seregni M, Paganelli C, Lee D, Greer PB, Baroni G, Keall PJ, et al. Motion prediction in MRI-guided radiotherapy based on interleaved orthogonal cine-MRI. Phys Med Biol. 2016;61(2):872-87.

18 van Sörnsen de Koste JR, Palacios MA, Bruynzeel AME, Slotman BJ, Senan S, Lagerwaard FJ. MR-guided gated stereotactic radiation therapy delivery for lung, adrenal, and pancreatic tumors: a geometric analysis. Int J Radiat Oncol Biol Phys. 2018;102(4):858-66.

19 Raaymakers BW, Jürgenliemk-Schulz IM, Bol GH, Glitzner M, Kotte ANTJ, Van Asselen B, et al. First patients treated with a 1.5 T MRI-Linac: clinical proof of concept of a high-precision, high-field MRI guided radiotherapy treatment. Phys Med Biol. 2017;62(23):L41-50. 